\title{
可见光驱使下有机染料与镍配合物催化放氢芳构化反应研究
}

\author{
王孝菊董奎刘强* \\ (兰州大学功能有机分子化学国家重点实验室 兰州 730000)
}

\begin{abstract}
摘要 在可见光催化下, 联合廉价易得的有机染料曙红 Y 与容易制备的镍催化剂在室温条件下, 高效绿色地实现了分 子内的脱氢芳构化, 此过程中只放出氢气无副产物, 无需外在氧化剂, 条件温和, 产率高, 对环境友好. 此外, 在其他 条件相同的情况下, 将绝氧条件下的放氢反应跟与有氧条件下的需氧氧化反应进行了对照, 发现汉斯酯衍生物及 $1,4-$ 二氢嘧啶衍生物在放氢条件下得到较高产率; 而在需氧氧化条件下 $3,4-$ 二氢噻吩衍生物、2,5-二氢吡咯衍生物芳构化的 转化率更高.
\end{abstract}

关键词 曙红 Y; 镍配合物; 放氢; 芳构化

\section{Visible-Light-Driven Aromatization Hydrogen Evolution by Organic Dye and Ni Complex}

\author{
Wang, Xiaoju Dong, Kui Liu, Qiang* \\ (State Key Laboratory of Applied Organic Chemistry, College of Chemistry and Chemical Engineering, Lanzhou University, \\ Lanzhou 730000)
}

\begin{abstract}
Pyridine derivatives play an important role in curing and controlling mites, bacteria, weed and so on. Pyrimidine derivatives exist in a number of bioactive natural products, and they have anti-allergy, anti-cancer, anti-inflammatory, insecticidal and some other properties. 3,4-Disubstituted thiophenes not only are important units for the synthesis of natural products, but also serve as key components in some biologically active compounds and material chemistry. In modern society, we have the urgent demand for achieving our products atom economicly and environment-friendly. Under this background, "atom-economy" reactions have been drawing great attention from many chemists and they have got many exciting improvements since then. So, we want to make our own contributions to this area and the following are some of our preliminary results. Our method was based on synergistic application of eosin Y with nickel (II) complex and an external oxidant-free oxidative dehydrogenation aromatization has been developed. At room temperature, Hantzsch 1,4-dihydropyridines, 1,4-dihydropyrimidines, 2,5-dihydrothiophenes and 2,5-dihydropyrroles were transformed into corresponding aromatic compounds in excellent yield under visible light irradiation via hydrogen evolution. We determined the hydrogen with GC-TCD using pure hydrogen as an external standard. It features very mild reaction conditions, high yields and excellent chemo-selectivity. In the previous reports, these transformations usually required higher temperatures and/or stronger oxidizing reagents, resulting in the generation of a large amount of by-products. In addition, the hydrogen evolution reactions were also compared with those of aerobic dehydrogenation. The results indicated that the dehydrogenation aromatizations of hantzsch 1,4-dihydropyridines and 1,4-dihydropyrimidine derivatives under the hydrogen evolution conditions proceeded in higher yields but very low conversions, while the reactions of 2,5-dihydrothiophenes and 2,5-dihydropyrroles gave higher conversions in the aerobic dehydrogenation conditions. So far, this is the first report using organic dye material combined with nickel (II) complexes to achieve dihydrogen dehydrogenation aromatization of heterocyclic compounds.

Keywords eosin-Y; nickel complex; hydrogen evolution; aromatization
\end{abstract}

\section{1 引言}

随着科技的进步和社会的发展，人类在获取更多自 然资源的同时，越来越注重将人类行为对环境的影响降 至最小. 在此背景下, 以 “原子经济性” 为原则的绿色 化学受到众多有机化学家的关注. “原子经济性” 反应
要求在化学反应中原料的原子以最大比例进入到产品 之中，从而避免了对不可再生资源的浪费，并从根本上 限制了废弃物的排放. 脱氢反应是典型的“原子经济性” 反应 ${ }^{[1]}$. 许多具有重要生理活性的杂环化合物都可以由 其二氢化合物脱氢得到. 例如汉斯酯及其衍生物脱氢芳 构化的产物——吡啶衍生物在除螨、杀菌、除草 ${ }^{[2]}$ 等方

* E-mail: liuqiang@1zu.edu.cn
Received August 18, 2016; published November 15, 2016.

Supporting information for this article is available free of charge via the Internet at http://sioc-journal.cn.

Project supported by the National Natural Science Foundation of China (Nos. 21572090, 21172102) and the Fundamental Research Funds for the Central Universities (No. lzujbky-2015-49).

项目受国家自然科学基金(Nos. 21572090 and 21172102)和中央高校基本科研基金(No. lzujbky-2015-49)资助. 
面有重要作用. 再如, 嘧啶类衍生物存在于一些生物活 性的天然产物，它具有抗过敏 ${ }^{[3]}$ 、抗癌 ${ }^{[4]}$ 、消炎 ${ }^{[5]}$ 、杀 虫 ${ }^{[6]}$ 等作用. 由于二氢嘧啶可以经 Biginelli 反应 ${ }^{[7]}$ 得到, 通过二氢嘧啶类衍生物氧化生成嘧啶的报道很多. 此 外, 还有其他一些可由其二氢化合物脱氢得到的重要芳 杂环化合物. 比如, 3,4-二取代的噻吩不仅是合成天然 产物的重要单元 ${ }^{[8]}$ 而且是一些具有生物活性化合物和材 料化学的关键组成部分 ${ }^{[9]}$. 吡咯是属于卟啉类化合物、 胆色素、辅酶、生物碱等 ${ }^{[10]}$ 的基本结构单元. 它的应用 也在制药及导电聚合物 ${ }^{[11]}$ 方面有所体现.

由相应的二氢化合物得到芳杂环化合物, 通常需要 较高的温度或较强的氧化剂, 在脱氢反应完成的同时, 经常伴随着大量副产物的产生. 例如汉斯酯的氧化, 使 用了诸如高锰酸钾、硝酸、硝酸铈铵、三氧化铬、PCC 等较强及过量的氧化剂 ${ }^{[12]}$, 造成了不同程度的浪费及 环境污染, 不符合现在绿色化学的理念. 相较于氧化剂 参与的脱氢反应, 放氢反应不但避免了因氧化剂介入而 产生的副反应, 而且将脱去的 “氢” 转化为氢气 $\left(\mathrm{H}_{2}\right)^{[13]}$ —— 一种具有很高的燃烧焓的可持续的清洁能 源. 早在 2004 年, 吴骊珠、佟振合等 ${ }^{[14]}$ 发现可见光照射 下, 平面四边形三联吡啶铂(II)配合物可以催化汉斯酯 及其衍生物脱氢芳构化, 副产物为氢气. 随后, 他们以 该铂配合物为光催化剂, 实现了 2,5 -二氢吡咯、2,5-二氢 噻吩等化合物的芳构化产氢, 为这些化合物的高效、绿 色合成提供了新途径.

考虑到贵金属配合物价格昂贵及可能对环境产生 较大污染, 选择高效、环保、廉价的催化体系来实现放 氢芳构化反应更加具有应用价值. 我们设想以常见的有 机染料曙红 eosin-Y 为光催化剂, 以简单的镍(II)配合 物 ${ }^{[15]}$ 为产氢催化剂, 在可见光照射下, 实现多种二氢杂 环化合物的放氢芳构化反应. 同时, 我们以可见光催化 下的需氧脱氢芳构化 ${ }^{[16]}$ 作为对照, 探讨了这两类反应 体系对不同类型的底物的优劣, 为发展高效、廉价、绿 色的可见光催化脱氢反应提供依据.

\section{2 结果与讨论}

\section{1 条件的选择}

汉斯酯(1a)为模型底物, 1a (0.1 mmol), eosin-Y (3 $\mathrm{mol} \%$ ), 镍催化剂(8 $\mathrm{mol} \%$ ) 用甲醇做溶剂, 绝氧条件下 $450 \mathrm{~nm}$ 的蓝色 $3 \mathrm{~W}$ LED 灯照射下反应 $24 \mathrm{~h}$, 得到产物 $2 \mathrm{a}(41 \%)$ 和氢气 $\left(\mathrm{H}_{2}\right)$. 首先, 我们对溶剂进行选择 (Entries 1 6), 从中可以看出在溶剂 $\mathrm{EtOH} / \mathrm{H}_{2} \mathrm{O}(V / V=$ $1: 1,4 \mathrm{~mL}$ ) 时得到较为理想的结果 $2 \mathbf{a}$ (Entry 6: 96\%). 其次, $\mathrm{EtOH} / \mathrm{H}_{2} \mathrm{O}(V / V=1: 1,4 \mathrm{~mL})$ 作溶剂, 对加入的碱 (1.2 equiv.)进行篎选(Entries 7 10), 发现在加入 $\mathrm{NaOAc}$ (1.2 equiv.)后得到理想结果 2a (Entry 10: 99\%), 然后, 对 $\mathrm{NaOAc}$ 的量进行选择, 当 $\mathrm{NaOAc}$ (2.0 equiv.)时, 产率
略有降低. 最后，控制实验(Entries 12 14)表明，只有 在存在 eosin-Y, 镍配合物还有光照的前提下才能使反 应高效进行。

表 1 反应条件的优化 ${ }^{a}$

Table 1 Optimization of the reaction conditions ${ }^{a}$<smiles>CCOC(=O)C1=C(C)NC(C)=C(C(=O)OCC)C1c1ccccc1</smiles><smiles>CCOC(=O)c1c(C)nc(C)c(C(=O)OCC)c1-c1ccccc1</smiles>

2a

\begin{tabular}{lllll}
\hline Entry & Solvent & Base & Conv./\% & Yield $\%$ \\
\hline 1 & $\mathrm{MeOH}$ & - & 41 & 100 \\
2 & $\mathrm{THF}$ & - & 9 & 100 \\
3 & $\mathrm{DMF}$ & - & 8 & 100 \\
4 & $\mathrm{CH} \mathrm{CN}^{\mathrm{C}}$ & - & 22 & 100 \\
5 & $\mathrm{CH}_{3} \mathrm{CN} / \mathrm{H}_{2} \mathrm{O}$ & - & 20 & 100 \\
6 & $\mathrm{EtOH} / \mathrm{H}_{2} \mathrm{O}$ & - & 96 & 100 \\
7 & $\mathrm{EtOH} / \mathrm{H}_{2} \mathrm{O}$ & $\mathrm{K}_{2} \mathrm{CO}_{3}$ & 30 & 100 \\
8 & $\mathrm{EtOH} / \mathrm{H}_{2} \mathrm{O}$ & $\mathrm{Na}_{2} \mathrm{CO}_{3}$ & 48 & 100 \\
9 & $\mathrm{EtOH} / \mathrm{H}_{2} \mathrm{O}$ & $\mathrm{Cs}_{2} \mathrm{CO}_{3}$ & 26 & 100 \\
10 & $\mathrm{EtOH} / \mathrm{H}_{2} \mathrm{O}$ & $\mathrm{NaOAc}$ & 100 & 99 \\
$11^{b}$ & $\mathrm{EtOH} / \mathrm{H}_{2} \mathrm{O}$ & $\mathrm{NaOAc}$ & 100 & 98 \\
$12^{c}$ & $\mathrm{EtOH} / \mathrm{H}_{2} \mathrm{O}$ & $\mathrm{NaOAc}^{d}$ & 14 & 100 \\
$13^{d}$ & $\mathrm{EtOH} / \mathrm{H}_{2} \mathrm{O}$ & $\mathrm{NaOAc}$ & & 0 \\
$14^{e}$ & $\mathrm{EtOH} / \mathrm{H}_{2} \mathrm{O}$ & $\mathrm{NaOAc}$ & & 0 \\
\hline
\end{tabular}

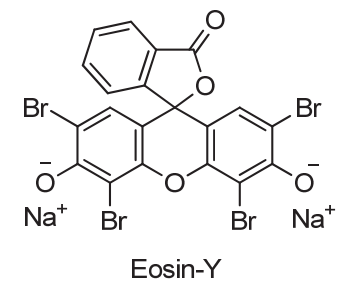<smiles></smiles>

${ }^{a}$ Reation conditions: Hantzsch ester (1a) $(0.1 \mathrm{mmol})$, EY (2 mg, 3 mol\%), Ni Complex (2.9 mg, $8 \mathrm{~mol} \%)$, base (1.2 equiv.) were added in solvent $(V / V=1$ : $1,4 \mathrm{~mL}$ ) under an argon atmosphere and irradiation of $3 \mathrm{~W}$ blue LEDs for $24 \mathrm{~h}$ at r.t. ${ }^{b} 2.0$ equiv. of base was used. ${ }^{c} \mathrm{Ni}$ complex was absent from the reaction system. ${ }^{d} \mathrm{EY}$ was absent from the reaction system. ${ }^{e}$ The reaction was carried out in the dark. 


\section{2 底物的适用范围}

\section{1 .1 底物扩展}

在已经优化了的条件下, 使用不同的 1,4-二氢吡啶 衍生物、1,4-二氢嘧啶衍生物、2,5-二氢噻吩衍生物和 2,5-二氢吡咯进行底物扩展. 实验结果如表 2 所示, 1,4二氢吡啶的衍生物 $(1 \mathrm{a}, 2 \mathrm{2})$ 在这一体系下转化率和产率 近乎完美, 都得到了几乎定量的产物. 1,4-二氢嘧啶衍 生物(3a)在这一体系下也有较好的结果, 得到了 $91 \%$ 的 产率(转化率为 $82 \%$ ), $4 \mathrm{a}$ 在这一体系中的产率为 74\%(转 化率为 $15 \%$ ), 不是很理想. 2,5 -二氢噻吩衍生物 $(\mathbf{5 a}, \mathbf{6 a})$ 在体系中的溶解性不理想, 因此加入了 $0.5 \mathrm{~mL}$ 的 THF 以增大溶解性. 这类底物产率很高, 但转化率较低. 类 似地, 2,5-二氢吡咯衍生物(7a)在较低的转化率下 $(56 \%)$ 得到了几乎定量产率的 $7 \mathbf{b}(99 \%)$. 可以看出, 该可见光 驱使的产氢体系对不同类型底物的脱氢反应, 均表现出 非常好的化学选择性, 但转化率因底物不同而改变.

表 2 芳构化底物的拓展 ${ }^{a}$

Table 2 Substrate scope of dehydrogenation aromatization

\begin{tabular}{llll}
\hline Entry & Substrate & Product & Conv. $/ \%$ Yield $/ \%$
\end{tabular}

1<smiles>CCOC(=O)C1=C(C)NC(C)=C(C(=O)OCC)C1c1ccccc1</smiles>

$1 a$<smiles>CCOC(=O)c1c(C)nc(C)c(C(=O)OCC)c1-c1ccccc1</smiles>

1b

2<smiles>CCOC(=O)C1=C(C)NC(C)=C(C(=O)OCC)C1c1ccc(OC)cc1</smiles>

2a<smiles>CCOC(=O)c1c(C)nc(C)c(C(=O)OCC)c1-c1ccc(OC)cc1</smiles>

2b

3<smiles>CCOC(=O)C1=C(C)NC(SC)=NC1c1ccccc1</smiles><smiles>CCOC(=O)c1c(C)nc(SC)nc1-c1ccccc1</smiles><smiles>CCOC(=O)C1=C(C)NC(SC)=NC1/C=C/c1ccccc1</smiles>

$4 a$<smiles>CCOC(=O)c1c(C)nc(SC)nc1/C=C/c1ccccc1</smiles>

4b
$100 \quad 99$

$82 \quad 91$
$15 \quad 74$

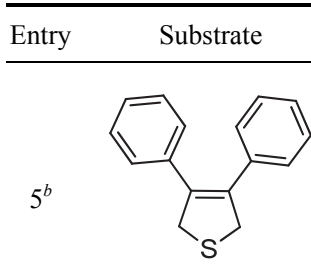

$5 a$
续表

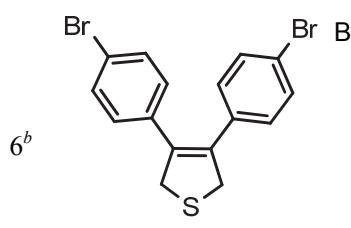

$6 a$<smiles>c1ccc(-c2cscc2-c2ccccc2)cc1</smiles>

$5 b$<smiles>C1=CCN(c2ccccc2)C1</smiles>

$7 a$<smiles>Fc1ccc(-c2cscc2-c2ccc(Br)cc2)cc1</smiles>

$6 b$<smiles>c1ccc(-n2cccc2)cc1</smiles>

$7 b$
${ }^{a}$ Unless otherwise stated, the reaction conditions are: substrates $(0.1 \mathrm{mmol})$, EY ( $2 \mathrm{mg}, 3 \mathrm{~mol} \%$ ), Ni Complex (2.9 mg, $8 \mathrm{mmol} \%$ ), base (1.2 equiv.) were added in solvent $(V / V=1: 1,4 \mathrm{~mL})$ under an argon atmosphere and irradiation of $3 \mathrm{~W}$ blue LEDs for $24 \mathrm{~h}$ at r.t.. ${ }^{b}$ With addition of $0.5 \mathrm{~mL}$ THF, NaOAc (1.2 equiv.) was used instead of $\mathrm{NaHCO}_{3}$.

\subsection{1 需氧脱氢芳构化作为对照实验}

和已经优化了的条件一致, 但不加入 2,4-二羟基苯 甲醛肟和镍(II)配合物, 而是在空气中用蓝色 $3 \mathrm{~W}$ LED 灯照射下，考察 1,4-二氢吡啶衍生物、1,4-二氢嘧啶衍生 物、3,4-二氢噻吩衍生物和 2,5 -二氢吡咯衍生物在反应 $24 \mathrm{~h}$ 后的脱氢芳构化转化率及产率. 实验结果如表 3 所 示, 1,4-二氢吡啶的衍生物 (1 a) 在这一体系下产率 93\%(转化率为 94\%). $2 \mathbf{a}$ 的产率为 $91 \%$ (转化率为 $88 \%$ ). 1,4-二氢嘧啶衍生物(3a)在这一体系下得到了 $62 \%$ 的产

表 3 在氧气条件下芳构化底物的拓展 ${ }^{a}$

Table 3 Substrate scope of dehydrogenation aromatization in air

\begin{tabular}{lllll}
\hline Entry & Substrate & Product & Conv./\% & Yield $/ \%$ \\
\hline 1 & $\mathbf{1 a}$ & $\mathbf{1 b}$ & 94 & 93 \\
2 & $\mathbf{2 a}$ & $\mathbf{2 b}$ & 88 & 91 \\
3 & $\mathbf{3 a}$ & $\mathbf{3 b}$ & 50 & 62 \\
4 & $\mathbf{4 a}$ & $\mathbf{4 b}$ & 34 & 61 \\
$5^{b}$ & $\mathbf{5 a}$ & $\mathbf{5 b}$ & 72 & 99 \\
$6^{b}$ & $\mathbf{6 a}$ & $\mathbf{6 b}$ & 58 & 98 \\
$7^{b}$ & $\mathbf{7 a}$ & $\mathbf{7 b}$ & 79 & 100 \\
\hline
\end{tabular}

${ }^{a}$ Unless otherwise stated, the reaction conditions are: substrates $(0.1 \mathrm{mmol})$, EY ( $2 \mathrm{mg}, 3 \mathrm{~mol} \%$ ), Ni Complex (2.9 mg, $8 \mathrm{mmol} \%$ ), base (1.2 equiv.) were added in solvent $(V / V=1: 1,4 \mathrm{~mL})$ under an argon atmosphere and irradiation of $3 \mathrm{~W}$ blue LEDs for $24 \mathrm{~h}$ at r.t.. ${ }^{b}$ With addition of $0.5 \mathrm{~mL}$ THF, NaOAc (1.2 equiv.) was used instead of $\mathrm{NaHCO}_{3}$. 
率(转化率为 50\%), 4a 在这一体系中的产率为 $61 \%$ (转化 率为 34\%). 2,5-二氢噻吩衍生物 (5a) 在体系中产率为 99\%(转化率为 $72 \%$ ), $6 \mathbf{a}$ 在体系中产率为 $98 \%$ (转化率为 $58 \%$ ). 2,5-二氢吡咯衍生物(7a)在这一体系下得到了(7b) 产率 $100 \%$ (转化率为 $79 \%$ ). 总体上说, 二氢噻吩衍生物 和二氢吡咯衍生物在光敏氧化条件下表现出比光催化 产氢条件下更好的转化率及产率.

\section{3 结论}

我们提出了一个在室温条件下通过可见光照射高 效催化二氢杂环化合物芳构化的方法: 结合廉价的有机 染料 Eosin-Y 与简单金属镍(II)配合物, 用乙醇和水作溶 剂, 在蓝色 $3 \mathrm{WLED}$ 灯照射下, 实现了分子内的放氢芳 构化反应. 该体系下大多数底物芳构化产率几乎为定 量, 显示了光催化放氢反应由于有效避免了外加氧化剂 引起的不必要的副反应, 产生了很高的化学选择性. 到 目前为止, 这也是首次使用有机染料与镍配合物相结合 使二氢杂环化合物脱氢芳构化的报道. 此外, 我们在其 他条件相同的情况下, 将绝氧条件的放氢反应跟在有氧 条件下的反应做了对比, 结果表明汉斯酯衍生物及 1,4二氢嘧啶衍生物在绝氧放氢反应条件下得到较高产率. 而在氧气条件下 2,5 -二氢噻吩衍生物、2,5-二氢吡咯衍 生物芳构化的转化率更高.

\section{References}

[1] (a) Li, Y. M.; Jia, F.; Ma, L. N.; Li, Z. P. Acta Chim. Sinica 2015, 73, 1311 (in Chinese). (李远明, 贾凡, 马丽娜, 李志平, 化学学报, 2015, 73, 1311.) (b) Xu, W. S.; Zhao, S. J.; Bi, X. H.; Liao, P. Q. Chin. J. Org. Chem. 2015, 35, 2095 (in Chinese). (徐文帅, 赵寿经, 毕锡和，廖沛球，有机化学， 2015，35，2095.) (c) Kone，J. R.; Marinescu, S. C.; Brunschwig, B. S.; Winkler, J. R.; Gray, H. B. Chem. Sci. 2014, 5, 865. (d) Li, Q. H.; Huang, R.; Wang, C. J. Acta Chim. Sinica 2014, 72, 830 (in Chinese). (李清华, 黄蓉, 王春江, 化学学报, 2014, 72, 830.) (e) Dobereiner, G. E.; Crabtree, R. H. Chem. Rev. 2010, 110, 681.

[2] Khadikar, B.; Borkat, S. Synth. Commun. 1998, 28, 207.

[3] Ban, M.; Taquchi, H.; Katsushima, T.; Akoki, S.; Wantanbe, A. Bioorg. Med. Chem. 1998, 6, 1057.
[4] Wright, G. E.; Gombino, J. J. J. Med. Chem. 1984, 27, 181.

[5] Jalander, L. F.; Longquist, J. E. Heterocycles 1998, 48, 743.

[6] Srivastva, S. K.; Agarwal, A.; Murthy, P. K.; Chauhan, P. M. S.; Agarwal, S. K.; Bhaduri, A. P.; Singh, S. N.; Fatima, N.; Chatterjee, R. K. J. Med. Chem. 1999, 42, 1667.

[7] (a) Kappe, C. O. Tetrahedron 1993, 49, 6937. (b) Kappe, C. O. Acc. Chem. Res. 2000, 33, 879. (c) Bose, D. S.; Fatima, L.; Mereyala, H. B. J. Org. Chem. 2003, 68,587 .

[8] (a) Gribble, W. G. In Comprehensive Heterocyclic Chemistry, Eds.: Katritzky, A. R.; Rees, C. W.; Scriven, E. F. V., Pergamon, Oxford, 1996. (b) Press, J. B. In The Chemistry of Heterocyclic Compounds: Thiophene and Its Derivatives, Ed.: Gronowitz, S., John Wiley \& Sons, Inc., New York, 1991.

[9] (a) Roncali, J. Chem. Rev. 1992, 92, 711. (b) Facchetti, A.; Yoon, M. H.; Marks, T. J. Adv. Mater. 2005, 17, 1705. (c) Rath, H.; Prabhuraja, V.; Chandrashekar, T. K.; Nag, N.; Goswami, D.; Joshi, B. S. Org. Lett. 2006, 8, 2325.

[10] (a) Jones, R. A.; Bean, G. P. The Chemistry of Pyrroles, Academic Press, London, 1977, p. 1. (b) Sundberg, R. J. In Comprehensive Heterocyclic Chemistry, Vol. 4, Eds.: Katritzky, A. R.; Rees, C. W., Pergamon Press, Oxford, 1984, p. 370. (c) Fan, H.; Peng, J.; Hamann, M. T.; Hu, J. F. Chem. Rev. 2008, 108, 264.

[11] (a) Fuerstner, A. Synlett 1999, 1523. (b) Higgins, S. J. Chem Soc. Rev. 1997, 26, 247. (c) McCullough, R. D.; Ewbank, P. C. In Handbook of Conducting Polymers, Eds.: Skotheim, T. A.; Elsenbaumer, R. L.; Reynolds, J. R., Dekker M., New York, 1998, Chapter 9.

[12] (a) Zhu, X. Q.; Zhao, B. J.; Cheng, J. P. J. Org. Chem. 2000, 65, 8158. (b) Bocker, R. H.; Guengerich, F. P. J. Med. Chem. 1986, 28 1596. (c) Ko, K. Y.; Kim, J. Y. Terahedorn Lett. 1999, 40, 3207. (d) Itoh, T.; Nagata, K.; Matsuya, Y.; Miyazaki, M.; Ohsawa, A. J. Org. Chem. 1997, 62, 3582.

[13] (a) Zhang, G. T.; Hu, X.; Chiang, C. W.; Yi, H.; Pei, P. K.; Singh, A K.; Lei, A. W. J. Am. Chem. Soc. 2016, 138, 12037. (b) Zhang, G. T.; Zhang, L. L.; Yi, H.; Luo, Y.; Qi, X. T.; Tung, C. H.; Wu, L. Z.; Lei, A. W. Chem. Commun. 2016, 52, 10407. (c) Zhang, G.; Liu, C.; Yi, H.; Meng, Q.; Bian, C.; Chen, H.; Jian, J. X.; Wu, L. Z.; Lei, A. W. J. Am. Chem. Soc. 2015, 137, 9273. (d) McKone, J. R.; Marinescu, S. C.; Brunschwig, B. S.; Winkler, J. R.; Gray, H. B. Chem. Sci. 2014, 5, 865. (e) Thoi, V. S.; Sun, Y. J.; Long, J. R.; Chang, C. J. Chem. Soc. Rev. 2013, 42, 2388. (f) Vincent, K. A.; Parkin, A.; Armstrong, F. A. Chem. Rev. 2007, 107, 4366.

[14] (a) Zhang, D.; Wu, L. Z.; Zhou, L.; Han, X.; Yang, Q. Z.; Zhang, L. P.; Tung, C. H. J. Am. Chem. Soc. 2004, 126, 3440. (b) Wang, D. H.; Peng, M. L.; Han, Y.; Chen, B.; Tung, C. H.; Wu, L. Z. Inorg. Chem. 2009, 49, 9995. (c) Chen, Y. Z.; Wang, D. H.; Chen, B.; Zhong, J. J.; Tung, C. H.; Wu, L. Z. J. Org. Chem. 2012, 77, 6773.

[15] Xu, Y.; Yin, X.; Huang, Y.; Du, P.; Zhang, B. Chem. Eur. J. 2015, 21,4571 .

[16] (a) Wang, L.; Ma, Z. G.; Wei, X. J.; Meng, Q. Y.; Yang, D. T.; Du, S. F.; Chen, Z. F.; Wu, L. Z.; Liu, Q. Green Chem. 2014, 16, 3752. (b) Wei, X.; Wang, L.; Jia, W.; Du, S.; Wu, L.; Liu, Q. Chin. J. Chem. 2014, 32, 1245 